\title{
« Ils bougent, ils bougent. . . mais jamais où il faut ! » \\ La construction des sens de la mobilité dans un contexte institutionnel de placement
}

\author{
"They're moving, they're moving. . . but never where they should!" \\ The everyday construction of mobility meanings in the institutional context of youth foster care
}

\section{Fleur Guy}

Reçu le 13 novembre 2013; accepté le 24 juin 2014

(C) IFSTTAR et Éditions NecPlus 2014

\begin{abstract}
Résumé L'adolescence correspond à une période d'acquisition progressive de l'autonomie de déplacement et de la mobilité. Alors que celle-ci s'effectue majoritairement dans le cadre familial, le contexte d'une prise en charge sociale fondée sur le retrait total ou partiel d'un mineur de son domicile familial interroge la construction du sens de la mobilité dans un contexte institutionnel particulier. Cet article s'intéresse aux valeurs et justifications de la mobilité, comprenant les déplacements des individus et leur capacité à réaliser des déplacements, en mettant en regard deux niveaux d'analyse : la société et l'institution. Les observations et entretiens réalisés dans quatre établissements permettent un double constat. Bien qu'elle constitue un impensé de la prise en charge, la mobilité se voit attribuer des sens divergents, en fonction notamment des déplacements qu'elle permet, de leur destination et de leur conformité à un ensemble de normes, conformément à ce que l'on observe au niveau de la société. Malgré cette ambivalence, un sens commun et partagé par les éducateurs et les adolescents est en construction au sein de l'institution.
\end{abstract}

Mots clés adolescent · compétence · mobilité · placement . système

Abstract Teenage years are often considered as a time of progressive learning of autonomous ability to selfdisplacement and mobility. While such learning mainly occurs in familial context, it can also take place outside the home, through institutional foster care. Foster care, defined as a social care for minor, out of their domestic sphere

Fleur Guy $(\bowtie)$

ENS de Lyon, Équipe Bio-géophile-UMR 5600 EVS, 15 parvis René Descartes, BP 7000, 69342, Lyon Cedex 07 France

e-mail : fleur.guy@univ-lyon2.fr for a given period, is a good viewpoint to question the construction of mobility's meanings. This article focuses on the values and justifications used to promote mobility at the society level and contrast them with everyday practices in specific institutional places. Based on ethnographic observations and interviews held in fours establishments, the argument is two-fold. First, although mobility is not explicitly a central value and objective in the educators' strategies, they practically confront different meanings of mobility when facing various problems, particularly the daily teenagers' travel. Second, beyond this heterogeneity of meanings, a common understanding of meaning is continuously negotiated and then shared among educators and teenagers.

Keywords foster care $\cdot$ teenager $\cdot$ mobility $\cdot$ skill $\cdot$ system

« Ils bougent, ils bougent » mais jamais où il faut ! » L'exclamation d'une éducatrice pendant une discussion informelle souligne les multiples possibilités d'interprétation du mouvement des adolescents dans le contexte du placement. Interrogée sur les déplacements des adolescents et sur leur capacité à utiliser les transports en commun, elle répond en prenant pour acquis le fait que les adolescents se déplacent et semble plus préoccupée par les lieux dont leurs déplacements permettent la fréquentation que par la capacité de déplacement en elle-même. Plus précisément, l'expression « jamais où il faut » suggère que les mouvements ne sont pas appropriés à la situation ou conformes à certains attendus, implicites ici. L'utilisation du verbe «falloir » renvoie ici à un devoir (qui n'est pas accompli) mais aussi à des conventions (qui ne sont pas respectées) et questionne donc les valeurs et normes associées à la mobilité. Enfin, l'utilisation du verbe «bouger» insiste sur le mouvement plutôt que sur la destination du déplacement et connote ce dernier négativement.

Le placement correspond à l'hébergement et la prise en charge d'un jeune hors de son domicile familial, pour une durée donnée. Il intervient dans le cadre d'une mesure 
administrative, judiciaire civile ou judiciaire pénale et s'inscrit globalement dans le champ de la Protection de l'enfance. Articulé en France autour de deux institutions, l'ASE (Aide sociale à l'enfance) et la PJJ (Protection judiciaire de la jeunesse), ce champ est marqué par l'oscillation entre les représentations d'un enfant coupable, dangereux, menaçant ou, au contraire victime, en danger, menacé. Les parallèles sont nombreux et leur utilisation pour des titres d'articles ou d'ouvrages $[1 ; 2 ; 3]$ révèle l'influence de ce débat dans la sphère scientifique comme professionnelle. Alors qu'on pourrait croire que ces deux positions opposées de l'enfance et de l'adolescence s'appliquent à des groupes d'individus distincts, elles peuvent caractériser une même personne, soumise à des procédures distinctes.

Cette prise en charge sociale a des implications fortes en termes de spatialité des adolescents. Comprise comme « la dimension spatiale de l'(inter)action des opérateurs d'une société », la spatialité se décline au niveau individuel puisque chaque «opérateur possède une spatialité propre, spécifique, construite dans l'action et qui articule des agencements spatiaux variés en un agencement englobant » [4]. En introduisant un nouveau lieu de vie pour les adolescents, la mesure de placement modifie cet agencement. Elle peut ainsi être considérée comme une forme de mobilité résidentielle qui modifie la mobilité quotidienne des adolescents concernés. Incluant des échelles temporelles multiples, la mobilité telle qu'elle est ici entendue comprend les déplacements effectifs des individus mais aussi la capacité à réaliser des déplacements, c'est-à-dire à réaliser « un mouvement d'un point à un autre dans l'espace, avec retour » ou de manière durable [5]. Cette dimension du concept de mobilité renvoie à la motilité, c'est-à-dire à « la manière dont un individu ou un groupe fait sien le champ du possible et en fait usage pour développer des projets » [6]. La possibilité même des déplacements dépend alors, d'une part, de l'accessibilité du lieu, d'autre part, des compétences des individus, à condition qu'il y ait connaissance de la première et mobilisation de la deuxième [6]. On s'intéresse donc ici aux ressorts de la mobilité et notamment aux différentes compétences permettant la réalisation du déplacement. Cet article envisage plus spécifiquement la mobilité quotidienne des individus mais s'appuie aussi sur une prise en compte plus large des « formes de mobilité, permettant de décrire des comportements divers où les rapports à l'espace-temps ne sont pas réductibles à une simple question d'échelle ou de fréquence » [7]. On distingue en outre ce qui relève de la mobilité comme potentialité, intégrant notamment les compétences et l'accessibilité, et ce qui relève des déplacements effectifs.

Si la mobilité est une notion polysémique dont on doit dans un premier temps préciser la signification, son sens, entendu comme la « raison d'être, valeur, finalité de quelque chose, ce qui le justifie et qui l'explique $»^{1}$, nécessite lui aussi des explications. Faisant l'objet d'un intérêt croissant en sciences sociales, la mobilité peut être considérée comme un nouveau paradigme [8], en lien avec celui de la modernité. Le double constat de l'avènement de la mobilité comme valeur des sociétés occidentales et des liens entre mobilité et rapports de pouvoir questionne ainsi le sens de la mobilité au niveau macro-social. L'intégration de la mobilité à la réflexion sur les rapports de pouvoir entre individus implique par exemple de considérer la mobilité géographique « comme une expression paradigmatique de la mobilité » [9]. La complexité de l'imbrication entre mobilité spatiale et mobilité sociale nécessite également de s'intéresser aux différents contextes sociaux de production de la mobilité spatiale et à l'impact de la mobilité spatiale sur ces contextes [10]. La place de la mobilité dans la société et plus précisément les liens entre mobilité et inégalités sociales sont en débat, mobilisant différents sens de la mobilité. Face à ce constat, Tim Cresswell note la «nécessité de considérer les mouvements de l'homme en relation avec les autres et d'être façonnés par des conventions et des institutions qui lui octroient un sens » [11]. Ainsi, le sens des mobilités peut être analysé depuis le point de vue des acteurs la réalisant [12] mais aussi depuis celui d'autres acteurs qui y contribuent par des incitations ou des restrictions. Dans ce contexte, cet article interroge la construction des sens de la mobilité dans le contexte institutionnel de placement. Comment la mobilité des adolescents est-elle interprétée par les adolescents qui la réalisent et par les éducateurs qui l'encadrent ? Comment ce sens se situe-t-il par rapport aux représentations sociales de la mobilité ?

Cet article présente dans un premier temps les différents contextes d'analyse des sens de la mobilité des adolescents placés. La mise en regard des représentations sociales associées à la mobilité avec une analyse centrée sur l'institution révèle dans un second temps des sens divergents, d'une part entre les différents niveaux (société/institution) et d'autre part entre les différents acteurs au sein de l'institution. Enfin, cette approche interroge dans un troisième temps la construction d'un sens commun et propre au contexte institutionnel.

Afin d'appréhender les variations autour des sens de la mobilité, cet article s'appuie sur une enquête menée dans quatre foyers du département du Rhône, entre octobre 2011 et juin 2013, avec des présences d'une à trois journées par semaine sur une période d'environ dix mois dans chaque établissement. Les quatre établissements de l'enquête ont été choisis en fonction de leur localisation, afin de prendre en compte le contexte territorial du lieu de placement dans les analyses. Ce contexte est défini par un ensemble de trois indicateurs : la catégorie de la commune du foyer selon le

\footnotetext{
${ }^{1}$ Dictionnaire Larousse.
} 
ZAUER (Zonage en Aires Urbaines et Aires d'Emploi de l'Espace Rural de l'Institut national de la statistique et des études économiques (INSEE), le temps de parcours depuis l'établissement à différents lieux en transport en commun, et les caractéristiques physiques de l'environnement immédiat des établissements. Trois types de localisation se dégagent ainsi : rurale (Foyer 1), périurbaine (Foyer 2) et urbaine (Foyers 3 et 4 ).

L'enquête repose sur une triangulation méthodologique, seul moyen de comprendre le rôle de la mobilité dans les jeux d'acteurs, dans les rapports à l'espace et plus globalement dans l'histoire de vie des jeunes. Une démarche ethnographique a tout d'abord conduit à l'observation de différentes situations et à la participation à certaines d'entre elles. Une attention particulière a été portée aux modalités de sorties des adolescents, dont différentes caractéristiques comme l'encadrement (présence/absence d'éducateur), la composition (seul/à plusieurs), la planification (prévue/improvisée) et la prescription (obligation/autorisation/interdiction) ont été notées. La participation aux sorties collectives avec des éducateurs mais aussi l'accompagnement d'un adolescent seul, visait à réaliser une « géographie en mouvement » [13]. À l'instar des migrations internationales étudiées par Julien Brachet, la mobilité quotidienne gagne ainsi à être étudiée via une observation elle-même mobile. Le fait d'être présente dans la voiture pendant les sorties avec les éducateurs a par exemple permis d'observer les choix d'itinéraires, les discussions qu'ils entraînaient, les discours associés aux passages dans tel ou tel lieu. Les sorties avec un seul adolescent ont également permis d'observer leur usage de l'espace et de construire « un terrain rhizomique en accordant a priori un potentiel heuristique identique aux points et aux lignes qui le constituent, c'est-à-dire aux localités et aux itinéraires » [13]. Les interactions entre acteurs éducatifs et institutionnels, en contexte formel (réunion) ou informel ont également été au centre de cette démarche d'observation, afin de considérer la part des interprétations individuelles au sein du dispositif institutionnel et d'en analyser certaines contradictions.

Dans le même temps, une série d'entretiens auprès de différents acteurs de la Protection de l'enfance a été réalisée. L'article s'appuie plus précisément sur une vingtaine d'entretiens avec des éducateurs et éducatrices des quatre foyers de l'enquête. Le choix d'interroger les éducateurs se justifie par leur présence quotidienne auprès des adolescents et par leur rôle privilégié dans la gestion de leurs déplacements. Les entretiens semi-directifs ont abordé plusieurs thématiques pour comprendre le rôle attribué à la mobilité dans la prise en charge ${ }^{2}$. Réalisés pendant les

\footnotetext{
${ }^{2}$ Les premières questions posées en entretien concernaient les personnes accueillies dans l'établissement (caractéristiques, raison du placement, provenance géographique). Ensuite, la question de la
}

périodes d'observations, les entretiens se sont également fondés sur certaines situations observées. Leur analyse s'attache au recueil d'informations factuelles et à un « travail interprétatif », passant par une attention portée aux mots et plus particulièrement au « langage indigène » [14]. Plus précisément, la manière dont les éducateurs parlent des adolescents, de leur rapport à l'espace et de leurs sorties, a retenu notre attention. Les termes utilisés en entretien sont alors mis en regard de ceux mobilisés officiellement par l'institution, dans les règlements, projets d'établissements et dans l'ensemble des interactions observées au cours de l'ethnographie. Enfin, l'article repose plus marginalement sur des entretiens réalisés avec les adolescents $(\mathrm{N}=45)$. Centrées sur leurs représentations spatiales, les questions posées concernaient également leurs conditions de mobilité.

Le matériau ainsi récolté doit être mis en relation avec les conditions même du recueil. L'entrée dans les établissements s'est faite par l'intermédiaire de leurs directions, suivie d'une présentation aux équipes après validation du projet. Bien que dans tous les établissements de bonnes conditions d'enquêtes aient été offertes, l'intégration aux équipes éducatives a dans certains cas été ressentie comme délicate, la méconnaissance du statut de chercheure et de doctorante et de la finalité du projet venant parfois se surajouter aux rapports hiérarchiques marqués entre personnels des établissements. De longs temps de présence dans les établissements et le partage d'horaires atypiques ont néanmoins permis d'instaurer une certaine proximité avec les équipes éducatives. Une phase de familiarisation avec les adolescents a également été nécessaire pour qu'ils identifient le statut chercheure et de doctorante et le différencie de celui d'une éducatrice ou d'une stagiaire-éducatrice. Certaines habitudes (renvoyer systématiquement vers un éducateur les adolescents pour toutes leurs demandes, refuser de rester seule sans éducateur au sein de l'établissement), avaient pour objectif d'affirmer une certaine indépendance vis-à-vis de l'institution pour que les adolescents acceptent de parler librement pendant les entretiens. Cette volonté d'indépendance dans le cadre d'une méthode ethnographique doit être nuancée car elle est dans certains cas difficilement tenable et questionne plus globalement l'implication du chercheur dans les faits qu'il observe [15].

mobilité quotidienne a été abordée à travers une série de questions relatives aux sorties à l'extérieur de l'établissement. Enfin, des questions sur le parcours professionnel et résidentiel des éducateurs ont également été intégrées à la grille d'entretien. 


\section{La mobilité quotidienne des adolescents placés, des sens en question à différents niveaux}

La construction des sens de la mobilité renvoie dans un premier temps à plusieurs contextes d'analyse compris ici grâce à une approche systémique. La construction des sens de la mobilité à l'échelle de la société implique de questionner la relation espace/société et plus particulièrement les différents modes de gestion de la mobilité des jeunes et leur justification.

\subsection{Une approche systémique de la mobilité des adolescents placés}

L'analyse du sens de la mobilité se fait ici en référence à une population qualifiée par son appartenance à un âge de la vie spécifique, l'adolescence. Le choix de ce terme s'explique tout d'abord par la volonté de s'écarter de la dichotomie entre enfant et jeune, et des représentations associées à ces termes dans le champ de la Protection de l'enfance. Les dispositifs étudiés ici font référence à la fois à l'enfance, dans le cas de l'ASE et à la jeunesse dans le cas de la PJJ. L'utilisation différenciée des termes ne renvoie pas spécifiquement à une limite d'âge biologique, puisqu'un individu du même âge peut être pris en charge par l'un ou l'autre des dispositifs, mais plus à des attributs sociaux, avec une opposition entre enfance en danger et jeunesse délinquante mettant en danger la société. Plutôt neutre par rapport à cette dichotomie, l'adolescence permet également de prendre en compte les dimensions développementales de l'individu puisque le concept renvoie « au processus individuel de croissance ou de développement » [16]. Cette conception d'un individu en cours de développement rejoint celle des institutions de placement elles-mêmes, qui ont pour objectifs de protéger l'individu mais aussi de le préparer à une sortie de prise en charge et à l'autonomie, au sens d'indépendance par rapport au soutien des tiers ou des pouvoirs publics. L'adolescence correspond en outre à une période d'acquisition progressive de l'autonomie de déplacement et permet donc d'observer les conditions d'acquisition de la mobilité. Plus largement, l'aptitude des adolescents à se déplacer seul ou en ayant recours à « l'auto-mobilité apparaît comme un élément constitutif de l'adolescence puisqu'elle lie le temps libre à des formes personnelles de découverte de soi » [17] et participe ainsi à l'émancipation caractéristique de l'adolescence. Plusieurs travaux s'intéressent à l'apprentissage de la mobilité dans le cadre familial en prenant en compte un ensemble de facteurs. Le «fonctionnement familial » influe ainsi sur l'acquisition de la mobilité, de même que le contexte résidentiel et les représentations qu'en ont les parents [18,
19, 20]. Le contexte résidentiel est dans d'autres cas pris en compte pour ses caractéristiques socio-économiques et permet d'analyser les mobilités adolescentes dans une dimension comparative [21].

À cette première spécificité liée à l'âge de la vie choisi dans le cadre de cette recherche, s'ajoutent les implications liées à la situation de placement. Cette mesure est fondée sur la séparation, plus ou moins temporaire, $\mathrm{du}$ jeune avec sa famille et implique donc une distance avec le domicile familial. Au-delà de cette définition, le placement renvoie à de multiples situations. Une typologie des motifs de placement permet dans un premier temps de clarifier les causes du placement à l'ASE à travers trois catégories : maltraitance, situation familiale et situation du jeune [22]. Au-delà de la différence liée au sexe, les filles étant significativement plus souvent placées pour des raisons de maltraitance que les garçons, les motifs d'entrée hors maltraitance sont soumis à l'âge de l'enfant : " Les prises en charge dans la petite enfance et dans l'enfance sont motivées par la situation familiale défaillante (voire absente) ; à partir de la pré-adolescence, ce sont les problèmes de comportement du jeune, des tensions avec sa famille (conflits familiaux) au point qu'une séparation physique s'impose » [22].

L'absence de famille peut également être un motif de prise en charge, notamment dans un contexte international. Suite à la ratification de la CIDE (Convention internationale des droits de l'enfant), la France prend en charge tous les mineurs présents sur son territoire et en situation de danger, sans distinction de nationalité. C'est sur ce principe que se fonde la prise en charge des Mineurs isolés étrangers, catégorie désignant « un étranger se disant mineur et qui se trouve sans représentant légal sur le territoire français » [23]. Enfin, le placement peut également intervenir dans le cadre de la justice pénale, suite à des infractions commises par l'adolescent et dans le cadre de l'Ordonnance du 2 février 1945 relative à l'enfance délinquante.

Si le cadre familial et le contexte résidentiel du domicile familial sont des éléments déterminants de l'acquisition de la mobilité pour les adolescents, la situation de placement nécessite de prendre en compte la prise en charge sociale dans ce processus. Le cas du placement des adolescents engage une spécificité théorique dans l'analyse des mobilités adolescentes et de leur apprentissage. Le placement correspond ainsi à une forme de mobilité résidentielle particulière, notamment du point de vue de l'intentionnalité du déplacement. Elle nous situe dans le contexte d'une mobilité spécifique, qui peut être contrainte. Du point de vue des spatialités, le placement entraîne parfois une situation de double, voire de multi- résidentialité pour les adolescents. En effet, ils conservent en général la possibilité de passer une à plusieurs nuits au domicile parental, le plus souvent le week-end. Inscrites dans l'ordonnance de placement, les 
modalités des retours en famille sont multiples et peuvent être très étendues dans le temps, notamment dans le cadre de certains placements dits séquentiels, où l'adolescent peut passer plus de temps au domicile parental qu'au foyer. Dans le cas de parents ne vivant pas ensemble, plusieurs domiciles peuvent être fréquentés au cours de ces retours en famille. Comme dans le cas du divorce des parents, le placement introduit une « incitation structurelle à la mobilité et donc l'acquisition de compétences de mobilité leur permettant de gérer cette spatialité familiale plus complexe » [19]. Si la situation des adolescents placés est complexe au regard de leurs lieux de résidence, il en va de même lorsqu'on envisage l'ensemble des institutions avec lesquelles ils sont en lien. En parallèle de l'inscription dans des dispositifs de droit commun, la fréquentation d'autres institutions spécialisées, pour la scolarité et l'insertion professionnelle (Institut thérapeutique éducatif et psychologique (ITEP) ou Maison familiale rurale (MFR) par exemple), en lien parfois avec le suivi judiciaire (Unité éducative d'activités de jour (UEAJ) ou autre services territoriaux éducatifs de milieu ouvert de la PJJ), entraîne des déplacements spécifiques pour certains adolescents.

La situation de placement semble ainsi multiplier les lieux pratiqués par les adolescents, du point de vue de la résidence (foyer et domicile familial dans la plupart des cas) comme des autres activités. Elle implique des agencements spatiaux mais aussi des modalités d'apprentissage de la mobilité spécifiques. Du fait de l'intervention d'acteurs institutionnels et éducatifs dans le quotidien des adolescents, le cadre familial ne suffit pas à rendre compte des conditions d'acquisition et de réalisation de la mobilité. Cette situation invite également à décentrer le regard par rapport à une mobilité qui permettrait aux adolescents de s'autonomiser par rapport aux parents et de découvrir des lieux inconnus [21]. Si cette approche s'applique aux adolescents en situation de placement, elle gagne à être complétée par une prise en compte de leur « habiter » qui considère la multiplicité des espaces dans lesquels les individus évoluent [4].

Pour cela, il est nécessaire de replacer la situation institutionnelle dans l'ensemble de systèmes où elle s'inscrit. L'approche systémique proposée par Urie Bronfenbrenner et son « modèle écologique de développement humain » permet de penser les relations complexes entre l'individu et différents cadres dans lequel il évolue, tout en prenant en compte le contexte social plus global [24]. Nous appuyons ainsi notre analyse sur l'identification de différents contextes de la mobilité et de leurs relations, selon les principes de l'écologie du développement humain définie par Urie Bronfenbrenner comme : the scientific study of the progressive, mutual accommodation, throughout the life span, between a growing human organism and the changing immediate environments in which it lives, as this process is affected by relations obtaining within and between these immediate settings, as well as the larger social context, both formal and informal, in which the settings are embedded $[24]^{3}$.

Centré sur l'individu en développement, ce modèle est particulièrement adapté à l'analyse du rapport à l'espace de l'adolescent. En outre, l'attention portée à différents cadres ${ }^{4}$ dans les relations qu'ils entretiennent entre eux et avec le contexte social nous permet d'envisager l'adolescent placé dans d'autres cadres que l'établissement. Ces cadres sont définis par un ensemble d'éléments : l'espace, le temps, les caractéristiques physiques, l'activité, les individus y participant et leur rôle [24]. À l'inverse d'une approche qui serait centrée sur l'établissement, les relations entre les différents cadres dans lesquels évoluent les adolescents, mais aussi entre les différents niveaux, du micro au macro, nous intéressent ici. Marqueur et condition de ces relations, la mobilité est analysée à différents niveaux identifiés dans cette approche, partant de l'environnement immédiat dans lequel évolue l'individu (microsystème) jusqu'au contexte social (macrosystème). Les processus sont ainsi analysés en considérant différentes échelles, allant du quotidien des individus, aux cadres d'organisation qui régissent et organisent ce quotidien, jusqu'au niveau plus idéologique. Ceci permet de considérer à la fois les systèmes normatifs et les cadres de pensée de la société sur un sujet donné, ici la mobilité.

Tout d'abord, l'établissement de placement peut être considéré comme un «microsystème » entendu comme le niveau le plus immédiat où se développent les relations de l'individu à l'environnement. À ce niveau, la mobilité découle en grande partie de la relation de l'individu à l'établissement et comprend aussi le sens de la mobilité pour les autres acteurs de l'institution, notamment les éducateurs. Ce niveau intègre l'ensemble de contraintes institutionnelles, écrites ou matérialisées, qui conditionnent la mobilité des adolescents. Le modèle de Bronfenbrenner permet d'associer plusieurs microsystèmes. En effet, dans le contexte d'une ouverture croissante des institutions de Protection de l'enfance sur l'extérieur, le foyer est un lieu pratiqué parmi d'autres. Ainsi, l'adolescent connaît d'autres cadres que le foyer. Le domicile familial ou l'établissement scolaire sont d'autres microsystèmes avec lesquels l'établissement de placement est en étroite relation. Le « mésosystème » renvoie alors aux relations entre les différents cadres qui contiennent la personne et la mobilité constitue un moyen de mettre en relation l'établissement

\footnotetext{
${ }^{3}$ L'étude scientifique des accommodements progressifs et réciproques, tout au long de la vie, entre un organisme humain en développement et son environnement immédiat en évolution constante, et de la manière dont ce processus est affecté par les relations qui règnent dans et entre ces cadres immédiats, ainsi que du contexte social plus large, formel ou informel, dans lequel les cadres sont contenus (traduction de l'auteur).

${ }^{4}$ Nous traduisons ainsi le terme de setting utilisé par Urie Bronfenbrenner.
} 
et les autres lieux de résidence ou de prise en charge. Cette dimension implique des paramètres extérieurs aux microsystèmes mais qui les influencent (l'« exosystème » dans l'approche d'Urie Bronfenbrenner). C'est dans ce cadre qu'intervient la notion d'accessibilité pour prendre en compte "l'offre de mobilité qui ne dépend pas des décisions de celui qui est mobile ou qui décide de la mobilité mais des autres composantes du système de déplacement qu'il utilise » [4]. Ces systèmes peuvent être définis comme des « infrastructures de la vie sociale [...] qui rendent le mouvement possible »[25]. Si on pense dans un premier temps au système de transports en commun, ce terme inclut également les dispostifs permettant la communication, comme le réseau de téléphonie mobile et l'ensemble des "systèmes numérisés interdépendants » [25]. Enfin, le « macrosystème » englobe les autres systèmes et se réfère aux systèmes sociaux, économiques, politiques ou juridiques notamment. Rapporté à la mobilité, ce système inclut les lois concernant la mobilité mais aussi les normes sociales qui lui sont associées ou encore les contraintes économiques qui la conditionnent, pour ne donner que quelques exemples.

\subsection{La mobilité comme valeur}

S'intéresser à la mobilité au prisme de ce modèle systémique implique dans un premier temps de comprendre les enjeux liés à la mobilité au niveau le plus englobant du système, et de s'intéresser aux représentations sociales de la mobilité. Conformément à la définition du sens retenue en introduction, les valeurs de la mobilité mais aussi la manière dont celle-ci est justifiée ou expliquée, interviennent à ce niveau.

L'importante place de la mobilité dans les sociétés occidentales, et même son caractère incontournable, sont dans un premier temps soulignés par de nombreux auteurs. Évoquant la mobilité comme «norme » [26], Jean-Pierre Orfeuil parle d'une «société de mobilité » [27], dans laquelle la mobilité est nécessaire à l'insertion des individus. L'idée d'une "mobilité généralisée », avancée par Pierre Lannoy et Thierry Ramadier s'appuie sur la mobilité comme « valeur dominante et transversale » dont le « caractère fondamentalement positif » fait consensus [28]. Les auteurs distinguent ainsi les " modalités de réalisation de cette mobilité » qui peuvent être critiquées et « le principe ou les processus, tant sociaux que cognitifs qui sous-tendent la mobilité [...]» et qui sont unanimement appréciés positivement [28]. Ces travaux nous situent ainsi dans une «société de la mobilité », issue des évolutions socio-économiques et technologiques ayant eu lieu depuis les Trente Glorieuses. Produite par la mobilité et l'essor de l'automobile, l'organisation actuelle de l'espace et de la société, et notamment la distance entre logements et emplois, serait ainsi fondamentalement marquée par la mobilité [27].

Cette nécessité se traduit par la mise en place de politiques publiques en faveur de la mobilité d'individus identifiés comme «pauvres » [29]. Le quartier de résidence est central dans cette identification puisque l'injonction à la mobilité s'appuie sur les conséquences négatives attribuées à la ségrégation, en termes d'accès à l'emploi et d'isolement social [29]. Parmi les « appartenances territoriales » retenues comme frein à la mobilité apparaissent ainsi « les territoires en politique de la ville », dont les habitants auraient une aptitude moins élevée à la mobilité [27]. Un « effet de quartier » influe alors ici sur les «manières d'habiter » des individus [30], dans une relation où l'ancrage et la faible mobilité ont une valeur négative. Plus globalement, la dualité entre immobilité et mobilité renvoie aux rapports de pouvoirs entre individus. Dans le « monde connexioniste » décrit par Luc Boltanski et Éve Chiapello, le «différentiel de mobilité » entre individus serait au fondement d'un nouveau mode d'exploitation [9]. Les auteurs démontrent ainsi que la capacité à user des différents réseaux des uns, et la stabilité des autres, s'inscrit dans un système dont les premiers profitent. Si les auteurs s'appuient sur une définition globale de la mobilité, la dimension spatiale sur laquelle ils insistent renvoie à une réflexion sur le rapport à l'espace comme capital des individus [31]. La notion de « capital spatial [définie comme] l'ensemble des ressources, accumulées par un acteur, lui permettant de tirer avantage en fonction de sa stratégie, de l'usage de la dimension spatiale de la société » [4] est discutée [32, 33]. Cette notion contribue en effet à isoler la composante spatiale des autres faits sociaux alors qu'il est possible de considérer la dimension spatiale des différents capitaux bourdieusiens $[32,33]$. La reconnaissance de la mobilité comme valeur de la société repose ainsi sur l'affirmation de liens causaux entre mobilité spatiale et mobilité sociale (et inversement entre immobilité spatiale et immobilité sociale). Les implications sociales de la mobilité pour les individus questionnent leur place dans la société et les modalités selon lesquelles ces places peuvent évoluer. Globalement définie comme un « espace et lieu où l'on se tient, la place signifie également par extension et abstraitement : position dans le monde, dans une collectivité » [5]. Le terme de place en anglais a le même sens, renvoyant à la location dans l'espace géographique mais aussi à la place dans la société [34]. Pour Michel Lussault, la place «met en relation, pour chaque individu, sa position sociale dans la société, les normes en matière d'affection et d'usage de l'espace en cours dans un groupe humain quelconque et les emplacements, [...] les endroits, que cet individu est susceptible d'occuper dans l'espace matériel en raison même de sa position sociale et des normes spatiales » 
[35]. Cette conception de la place met l'accent sur son aspect relationnel, sur les interactions entre espace matériel, norme sociale et position, qui contribuent à déterminer les « endroits» que les individus peuvent occuper. Ce système de relations complexes est également mis en évidence dans l'analyse des liens entre mobilité et inégalités sociales : la mobilité permet l'accès aux ressources offertes par différents espaces mais elle est en même temps conditionnée par un certain nombre de compétences physiques et cognitives. Les relations entre mobilité et inégalités sociales sont alors complexes : la mobilité peut être identifiée comme un facteur d'inégalités sociales, tout en étant elle-même influencée par ces inégalités [32].

Les liens entre mobilité sociale et spatiale sont discutés dans plusieurs travaux [29, 36, 37]. Le double système d'un « cercle vicieux de l'immobilité excluant et de celui du cercle vertueux de la mobilité intégratrice » peut être nuancé [36]. La relation entre exclusion sociale et immobilité peut tout d'abord être remise en cause puisque plusieurs analyses montrent que des populations précaires ou pauvres peuvent être mobiles. C'est le cas des précaires étudiés par Yves Jouffe, dont la fragilité économique entraîne une mobilité flexible qui peut être intense [36]. $\mathrm{Si}$ on s'intéresse plus particulièrement aux individus jeunes, et notamment aux adolescents, on peut également relativiser le lien entre immobilité et ségrégation sociale. Analysant ce lien, Nicolas Oppenchaim met en regard les mobilités quotidiennes d'adolescents vivant en Zones urbaines sensibles (ZUS) avec les pratiques d'adolescents vivant hors de ces zones. Moins accompagnés par leurs parents dans leurs déplacements, les adolescents vivant en ZUS fréquentent des espaces plus proches de leur domicile que ceux vivant dans d'autres quartiers [21]. Cependant, la prise en compte des seuls déplacements autonomes montre que les garçons vivant en ZUS sont caractérisés par « une autonomie et un usage des transports en commun plus précoce » que les adolescents vivant dans d'autres types de quartiers, ouvriers et moyens [21]. Le fait d'être moins accompagnés par leurs parents dans leurs déplacements semble limiter les distances parcourues par les adolescents vivant en ZUS, tout en favorisant un apprentissage précoce de la mobilité autonome pour les garçons [21].

Cette remise en cause de la relation univoque entre exclusion sociale et immobilité se double d'une remise en cause de la relation entre mobilité et insertion. En effet, des rapports à l'espace tendant vers l'immobilité peuvent être supports de ressources pour des populations pauvres. À travers deux enquêtes menées en France et aux États-Unis dans des quartiers pauvres, Sylvie Fol montre comment le quartier est un « lieu ressource » pour ses habitants [37]. Le recours par les ménages pauvres périurbains français aux ressources présentes dans le territoire local plutôt qu'à la motorisation relève également d'une adaptation aux contraintes de pauvreté qui passe par une « dépendance locale » plutôt que par une « dépendance automobile » [38]. S'appuyant sur les travaux de Jean-Noël Rétière [39], plusieurs chercheurs utilisent la notion de «capital d'autochtonie » pour mettre en évidence « les ressources que procure l'appartenance à des réseaux de relations localisés ", notamment pour les classes populaires [40]. Toujours dans un contexte rural, la faiblesse de la mobilité des allocataires du RMI peut être comprise comme une « adaptation des populations socialement disqualifiées à un contexte de disqualification spatiale » [41]. Enfin, d'autres exemples montrent que la mobilité spatiale n'est pas toujours associée à une mobilité sociale ascendante. Le suivi par Cécile Vignal d'une cohorte d'ouvriers ayant subi la relocalisation de leur entreprise relativise le parallélisme entre mobilité géographique et mobilité sociale et invite au contraire à prendre en compte " les coûts sociaux et la dimension humaine qu'impliquent ces formes de mobilité et de flexibilité de l'emploi » [42].

L'affirmation de la mobilité comme valeur de la société repose sur les liens de causalité entre la capacité de déplacement des individus et leur possible ascension sociale. Si la complexité des relations entre mobilité et inégalités sociales est dans un premier temps interrogée, la question de la finalité de la mobilité invite également à nuancer les représentations sociales associées à la mobilité.

\subsection{La mobilité comme norme : de la compétence à favoriser au risque à contrôler}

Le sens, dans la définition qui en a été retenue ici, fait intervenir la valeur, la justification, mais aussi la finalité de quelque chose. Cette dernière dimension de la définition a une portée heuristique importante : en replaçant la mobilité dans un ensemble d'objectifs, elle permet de nuancer les représentations sociales qui lui sont associées. Les deux exemples suivants montrent notamment que la perception de la mobilité des adolescents n'est pas uniforme.

Fondées sur les relations entre mobilité et ascension sociale, les politiques en faveur de la mobilité concernent des populations aux caractéristiques sociales spécifiques, et notamment les adolescents. Alors que la catégorie de jeunes, renvoyant à une tranche d'âge plus large, est souvent employée dans la littérature, les processus analysés concernent également les adolescents. Les jeunes figurent parmi les quatre groupes de population « fragilisés par les transformations actuelles de la société » [43]. Comme les femmes isolées avec enfants, les personnes issues de l'immigration et les hommes aux faibles niveaux de qualification, les jeunes peuvent être qualifiés d' « insulaires » de par leur rapport spécifique au territoire et à la mobilité [43]. Non seulement les jeunes correspondent à une catégorie 
de population particulièrement touchée par la précarité, mais l'état même de jeunesse fait de la mobilité un enjeu important à ce moment de la vie. L'étape de formation à laquelle correspond la jeunesse implique en effet un rapport spécifique au territoire qui pourrait avoir un rôle à jouer dans la construction de soi [43]. Plus généralement, c'est en lien avec un impératif d'insertion socioprofessionnelle que le manque de mobilité des jeunes pose problème. Alors qu'elle est l'objet de plusieurs dispositifs d'aide, la mobilité se voit attribuer des valeurs négatives et prend dans certains contextes un tout autre sens que celui d'une compétence nécessaire à l'insertion sociale.

Le pôle mobilité mis en place par la fédération des maisons de quartier de la ville de Saint-Nazaire est un exemple de dispositif public en faveur de la mobilité [44]. Les multiples actions mises en place dans ce cadre (location de cyclomoteur, apprentissage de la mobilité, système de taxi pour répondre aux horaires décalés, par exemple) ont ainsi pour objectif de favoriser la mobilité. Alors qu'elle cite la défiance à laquelle les jeunes doivent faire face comme une difficulté pour leur mobilité, Mélanie Gachelin évoque le rapport des jeunes à l'automobile en termes de risque et de danger : " Est-ce que les jeunes en difficulté sont des conducteurs plus dangereux que les autres ? Certainement et malheureusement. Car ils sont nombreux à avoir des conduites à risque, en particulier la consommation d'alcool et de cannabis. Même au nom de l'insertion et de l'égalité des chances, il n'est pas possible de transiger sur la sécurité » [44].

Ainsi, si on attend des jeunes en difficulté qu'ils soient mobiles, cette mobilité est par ailleurs appréhendée comme un risque. L'attribution de pratiques déviantes aux individus concernés justifie cette crainte face à leur mobilité. Deux ensembles de valeurs semblent alors s'opposer : "l'égalité des chances » et «l'insertion » d'un côté, et la « sécurité » de l'autre. On voit alors apparaître une certaine ambivalence par rapport à la gestion de la mobilité des jeunes, qu'il faut en même temps favoriser et contrôler.

Cette ambivalence apparaît également dans la perception de la mobilité d'autres jeunes. Travaillant sur " la catégorie des jeunes en errance », Djemila Zeneidi évoque une «mobilité dévalorisée »[45]. Son analyse de la construction idéologique de cette catégorie d'une part, et la retranscription du sens attribué à cette mobilité par les jeunes eux-mêmes de l'autre, révèle « une mobilité en décalage par rapport aux logiques sociales en vigueur mais qui n'en est pas moins nourrie par une capacité d'appropriation » de l'espace et des repères pour les jeunes en question [45]. On retrouve alors une forme d' « extrême mobilité » associée à des valeurs négatives, comme dans le cas du vagabondage ou de la migration [46].

Les représentations sociales associées à la mobilité révèlent des tensions entre différentes valeurs. La valeur de la mobilité semble dépendre du déplacement qui y est associé, et de sa conformité aux normes en vigueur. La prise en compte de la mobilité dans l'analyse des processus sociaux fait l'objet de controverses, concernant notamment le rôle de la mobilité ou de son absence dans la reproduction des inégalités sociales. Si les adolescents et les jeunes peuvent être concernés par ce défaut de mobilité, ce dernier correspond autant à un manque de mobilité qu'à une mobilité inadaptée par rapport aux normes sociales. Leur future insertion étant un des objectifs affiché du placement des adolescents, comment la mobilité est-elle intégrée à cette prise en charge et comment ces sens pluriels de la mobilité se déclinent-ils dans le contexte institutionnel du placement des adolescents?

\section{Les sens de la mobilité en contexte de placement : des approches divergentes}

À l'échelle de la société, la mobilité est considérée comme un moyen nécessaire, voire indispensable pour accéder à l'insertion sociale. Si celle-ci doit conduire à des comportements conformes aux normes sociales en vigueur pour être appréciée positivement, elle n'en reste pas moins un objectif affiché des politiques publiques à destination des adolescents. Dans le contexte du placement, la mobilité est peu objectivée par les éducateurs et relève de l'implicite. Malgré l'absence de référence explicite à la mobilité dans sa globalité, certaines de ses composantes font l'objet d'une attention particulière de la part des éducateurs. Le franchissement de la limite entre l'intérieur et l'extérieur est ainsi central à l'échelle micro. Si le passage à l'extérieur de l'établissement est fortement contrôlé et réglementé, le sens attribué au déplacement dépend largement de sa destination et des représentations qui lui sont associées.

\subsection{La mobilité, impensée du placement}

La mobilité dans le contexte de placement ne fait pas l'objet de réflexions spécifiques et n'est pas pensée en tant que telle par les équipes éducatives et acteurs institutionnels. Les entretiens réalisés avec les jeunes et avec les éducateurs révèlent l'absence de réflexion spécifique sur la mobilité.

Répondant à une question sur le rôle de la mobilité dans la prise en charge, un éducateur répond ainsi :

La notion de sens de l'orientation c'est pas..., ça fait pas sens au niveau éducatif, c'est pas quelque chose qui me tourmente. C'est-à-dire qu'il y a beaucoup d'autres trucs qui sont bien plus prioritaires! (Entretien, éducateur Foyer 1, 2012)

Cette remarque est intéressante à deux titres : d'abord dans la définition même de la mobilité, ici confondue avec « la 
notion de sens de l'orientation ». Si elle rejoint la mobilité comme compétence, cette conception de la mobilité est plutôt restrictive. D'autre part, la mobilité comme potentiel ressort de l'action éducative est ici disqualifiée, en invoquant l'absence de sens et de légitimité de cette question. Celle-ci se vérifie dans les pratiques des éducateurs. L'observation de situations précédant les sorties a également mis en évidence l'absence de protocole spécifique concernant les déplacements à l'extérieur. La préparation de la sortie se fait au cas par cas. Les entretiens avec les adolescents révèlent cette diversité de pratiques : certains expliquent avoir été accompagnés dans leurs déplacements par les éducateurs, puis par des pairs, avant de sortir seuls. Pour d'autres, même nouvellement arrivés dans la ville ou le pays, l'apprentissage de l'espace et des moyens de s'y déplacer s'effectue souvent avec l'aide des pairs. Plusieurs adolescents nouvellement arrivés à Lyon ou même en France expliquent ainsi que c'est grâce à la fréquentation d'autres adolescents, présents depuis plus longtemps, qu'ils ont apprivoisé la ville et les transports :

\section{Comment ça s'est passé à ton arrivée ?}

C'était difficile, je sortais pas, je connaissais pas l'endroit. . .

Et comment tu as fait pour apprendre à le connaître ?

Avec M, elle était arrivée avant, elle connaît l'endroit.

(Entretien, adolescente Foyer 3, 2012)

Est-ce que tu as des difficultés avec les transports?

C'est pas dur, si j'ai besoin je demande au conducteur. Au début je sortais pas, seulement avec les filles qui étaient là, pendant 3 mois je repérais pas les lieux, puis a commencé l'école...

(Entretien, adolescente Foyer 3, 2012)

Ces observations correspondent à une tendance générale mais doivent être relativisées par certains cas où la sortie est préparée, avec l'aide de différents supports (plans, itinéraires internet). La prévision d'un déplacement autonome peut ainsi faire l'objet d'un accompagnement préalable pour que l'éducateur montre le trajet à l'adolescent. C'est particulièrement le cas lorsque l'objet du déplacement est jugé important (santé, administratif. . .) et dépend également de l'évaluation de la capacité de l'adolescent à se déplacer. Lors de nos observations, un déplacement en lien avec des démarches administratives fait ainsi l'objet d'une préparation par l'éducatrice référente de l'adolescent. Plusieurs jours avant le rendez-vous en question, celle-ci réfléchit à l'itinéraire le plus adapté à l'adolescent, privilégiant le bus plutôt que le métro. Elle explique ce choix par la connaissance du réseau qu'a le jeune :

[...] parce qu'il ne connaît pas. Si il connaissait, il pourrait prendre le métro... mais il va se perdre, même si tu lui expliques, il va se perdre, vaut mieux qu'il prenne le bus. (Carnet de terrain, Foyer 4, 2013)

L'éducatrice imprime ensuite l'itinéraire et les horaires et précise qu'elle va aller avec lui le lendemain sur le lieu du rendez-vous, « en voiture, pour qu'il visualise ». On voit apparaître dans cet exemple l'intervention de la capacité de déplacement des adolescents dans la manière dont les éducateurs gèrent leur déplacement. Dans cet exemple, la sortie est préparée mais l'acquisition de compétences spatiales n'est pas centrale dans la démarche. L'étape de recherche d'informations sur internet ne fait pas l'objet d'un travail pédagogique et le repérage préalable est centré sur le lieu de rendez-vous plutôt que sur le trajet puisqu'il se fait en voiture.

L'usage de la voiture est en effet privilégié lorsqu'il s'agit d'accompagner un adolescent en déplacement. L'absence de possession du permis de conduire par les éducateurs constitue ainsi une forte contrainte, limitant les possibilités d'accompagnement, notamment en milieu rural. Il est à ce titre intéressant de noter que l'apprentissage de la mobilité peut être évoqué comme un argument en faveur de l'usage des transports en commun par des éducateurs n'ayant pas le permis de conduire. La mobilité comme compétence et sa nécessaire acquisition par les adolescents est donc évoquée mais plutôt par défaut, pour atténuer la contrainte liée à l'absence de permis de conduire.

Enfin, c'est à partir de cas particuliers d'adolescents pour lesquels les difficultés de mobilité sont identifiées que la mobilité semble prendre du sens pour les éducateurs. Ainsi, les éducateurs ont souvent répondu aux questions concernant le rôle de la mobilité dans la prise en charge en faisant référence à des adolescents ayant des difficultés à se repérer et se déplacer dans l'espace. La nécessité d'un accompagnement spécifique dans ces cas faisant figure d'exception est signalée par plusieurs éducateurs. Le cas de $\mathrm{K}$. a ainsi été cité par tous les éducateurs interrogés dans le foyer où elle est placée. Conscients de la problématique spécifique de cette jeune fille qui a du mal à se repérer dans l'espace et à sortir seule, ils font également part des difficultés de gestion de la prise en charge qu'elle entraîne. Liées à un manque de moyens humains (il faudrait pouvoir accompagner $\mathrm{K}$. dans ses déplacements pour lui apprendre les trajets et la rassurer), ces difficultés peuvent également s'expliquer par la faible prise en compte de cet enjeu dans la prise en charge éducative habituelle.

La faible prise en compte de la mobilité comme compétence à favoriser, présente dans le discours des éducateurs comme dans celui des adolescents, montre une première divergence entre le sens objectivé (la mobilité comme compétence à favoriser en vue d'une insertion) et le sens conféré par les acteurs, ici les éducateurs. Alors même que certains adolescents sont placés en raison de 
difficultés sociales, la mobilité n'est pas citée spontanément en entretien comme un facteur de ces difficultés ou comme un moyen d'insertion. Valorisée lorsqu'on se réfère au macrosytème, la mobilité constitue un impensé au niveau $\mathrm{du}$ microsystème. Une première discontinuité entre les sens de la mobilité observés dans les différents cadres proposés par le modèle de Bronfenbrenner peut ainsi être soulignée. Ce décalage peut être expliqué par la présence d'autres domaines identifiés comme prioritaires, tels que la scolarité et la formation, ou encore la santé. Il est sans doute aussi lié au fait que les éducateurs n'identifient pas de difficultés spécifiques concernant la capacité des adolescents à se déplacer, à l'exception de quelques cas particuliers. L'enquête de terrain contribue ainsi à mettre en lumière cet impensé du placement, notamment à travers la manière dont les déplacements quotidiens sont organisés et gérés dans les établissements.

\subsection{La gestion quotidienne des déplacements : une gestion de la mobilité ?}

Alors que la mobilité n'est pas énoncée comme une priorité éducative par les éducateurs, les observations réalisées dans les établissements révèlent la place centrale de la gestion des déplacements quotidiens. Le franchissement de la limite entre l'intérieur et l'extérieur de l'établissement est bien sûr en jeu dans cette gestion. Cependant, la gestion des déplacements quotidiens ne se limite pas à la sortie de l'établissement et implique une prise en compte de la mobilité des adolescents.

Les pratiques des jeunes sont fortement conditionnées par le placement. Dans le cas d'un placement judiciaire, l'ordonnance de placement prévoit la fréquence et les modalités de retour, plus ou moins régulier, au domicile familial et peut également indiquer certaines interdictions particulières. Les adolescents placés sont ainsi généralement autorisés à rentrer dans leur famille le week-end ou un week-end sur deux, mais le droit de visite ou d'hébergement au domicile familial n'est pas automatique. Dans le cas d'un placement administratif, un calendrier de sorties est également mis en place avec le service de l'ASE pour organiser les retours en famille de l'adolescent. Au-delà de ces prescriptions générales, l'adolescent placé est soumis au règlement intérieur de l'établissement qui pose le cadre des sorties à l'extérieur. Au cadre temporel de la sortie sans adulte, souvent autorisée entre le repas de midi et le repas du soir, s'ajoutent les modalités de la demande de sortie toujours soumise à l'acceptation de l'éducateur et matérialisée dans certains cas par un bon de sortie remis à l'adolescent ou l'annotation d'une feuille de sortie restant dans le bureau des éducateurs. Les règles concernant les sorties sont en général communes à l'ensemble des jeunes $\mathrm{du}$ foyer, mais une adaptation pour les adolescents ayant entre 16 et 18 ans, et plus de 18 ans, peut parfois leur permettre de sortir plus tard le soir.

Le contrôle humain du respect du règlement intérieur est renforcé dans certains cas par un dispositif spatial de contrôle des liens entre l'intérieur et l'extérieur de l'établissement. À l'inverse d'autres structures de la Protection de l'enfance, les foyers ne sont pas fondés sur la fermeture. Cependant, l'analyse de leur organisation spatiale permet d'identifier différents degrés d'enfermement en prenant en compte la discontinuité entre l'intérieur et l'extérieur de l'établissement (présence d'une enceinte encerclant l'ensemble de l'établissement, faiblesse des contacts directs avec l'extérieur de l'établissement) et la présence de dispositifs spatiaux de contrôle des circulations dans l'établissement et entre l'intérieur et l'extérieur de l'établissement (le choix de la position $\mathrm{du}$ bureau des éducateurs en fonction des possibilités de contrôle visuel des sorties, le contrôle systématique des entrées et sorties de l'établissement, la présence d'un système de surveillance électronique au sein de l'établissement). Un premier établissement correspond au plus fort degré d'enfermement. Il possède une enceinte encerclant l'ensemble de l'établissement, matérialisée par des murs associés en partie à des barbelés et palissades renforçant l'effet de clôture. Le dispositif de fermeture est complété par un portail systématiquement fermé, dont l'ouverture est commandée manuellement, après activation depuis le bureau des éducateurs. Un visiophone placé dans ce bureau permet également de contrôler les entrées dans le foyer. L'ordonnancement des lieux au sein de l'établissement répond au même objectif : la localisation du bureau et la présence de grandes fenêtres permettent aussi aux éducateurs d'observer les allers et venues dans l'établissement.

À l'inverse, deux autres établissements sont caractérisés par l'absence d'enceinte. Dans ces établissements, un contrôle systématique des entrées et sorties est rendu impossible par l'absence de portail ou sa facilité d'ouverture. Dans les deux cas, la localisation du bureau des éducateurs ne permet pas de contrôler les allers et venues. En outre, un des établissements a une façade sur rue et certaines salles à manger et leurs fenêtres, situées en rez-de-chaussée, donnent donc directement sur la rue. Cette disposition facilite les contacts avec l'extérieur, qu'ils soient visuels, sonores ou physiques. Il faut cependant relativiser cette absence de contrôle puisque l'un des établissements dispose d'une caméra de vidéosurveillance positionnée devant le portail. Enfin, un quatrième établissement se trouve dans une situation intermédiaire. En effet, la question de la fermeture du portail est en discussion pendant notre présence dans cet établissement.

Ces différents degrés d'enfermement dans l'organisation spatiale des établissements peuvent être mis en relation 
avec leurs localisations et avec la distance à certains lieux privilégiés de sociabilité adolescente. Les plus forts degrés d'enfermement et de distance ne se cumulent pas au sein des mêmes établissements : le Foyer 1, établissement le plus distant, est aussi le plus ouvert. Inversement, le Foyer 3 possède le plus grand degré de fermeture mais le plus faible concernant la distance. Enfin, le Foyer 2 se trouve en situation intermédiaire pour les deux modalités. Ces constats vont dans le sens d'une articulation des modalités de contrainte spatiale, et d'une substitution possible entre la mise à distance et l'enfermement. Cependant, le cas du Foyer 4, à la fois le moins distant et le moins fermé, montre que l'ouverture des établissements n'est pas propre au milieu rural et relativise l'hypothèse d'une complémentarité complète entre les deux modalités. Dans tous les cas, même si l'organisation spatiale de l'établissement et/ou sa localisation limitent les sorties à l'extérieur, celles-ci sont possibles, qu'elles soient autorisées ou non.

À partir de ce cadre réglementaire et spatial, les déplacements quotidiens font l'objet de nombreuses discussions entre éducateurs. Celles-ci révèlent une gestion différenciée des déplacements selon différents facteurs, et notamment la mobilité des adolescents. La prévision des déplacements se fait à l'occasion de réunions hebdomadaires au cours desquelles un éducateur est chargé de l'agenda. L'énoncé des différents rendez-vous prévus pour les adolescents au cours de la semaine à venir donne lieu à des discussions sur l'accompagnement ou non de l'adolescent. Celui-ci dépend bien sûr de la nature du rendez-vous mais aussi de son accessibilité et de la capacité de l'adolescent à se déplacer. L'âge des adolescents intervient par exemple comme argument dans les discussions concernant les sorties autonomes.

Les choix effectués concernant l'accompagnement ou non des déplacements dépendent ainsi d'un arbitrage entre impératifs organisationnels et évaluation de la capacité de l'adolescent à se déplacer seul. Les éducateurs doivent faire avec la mobilité des adolescents. Que leur capacité de déplacement soit limitée ou au contraire développée, la mobilité peut constituer une ressource ou une contrainte dans la prise en charge. Une mobilité limitée implique la présence d'un adulte pour tout déplacement et est donc source de difficultés d'organisation en même temps qu'elle assure l'éducateur de la présence de l'adolescent dans l'établissement et facilite la surveillance. Une mobilité développée des adolescents libère des effectifs de personnel lorsque l'adolescent doit se déplacer mais nécessite une surveillance accrue des entrées et sorties de l'établissement. La mobilité comme ressource ou contrainte de la prise en charge dépend en fait de la relation entre l'éducateur et l'adolescent, fondée sur la surveillance ou l'autonomie.

La gestion quotidienne des déplacements des adolescents relève bien d'une gestion de leur mobilité. Liée à des contraintes organisationnelles et l'évaluation des capacités de l'adolescent à se déplacer seul, celle-ci dépend aussi des représentations des lieux que les adolescents sont supposés fréquenter et implique une réflexion sur le rapport des éducateurs à l'espace.

\subsection{Le sens du déplacement : impact de la destination supposée du déplacement sur les sens de la mobilité}

La gestion des sorties à l'extérieur de l'établissement intègre une prise en compte des capacités de déplacement des adolescents avec lesquelles les éducateurs doivent composer. Cette gestion des sorties à l'extérieur ne peut cependant pas se comprendre en seule référence à l'établissement et ses limites avec l'extérieur. Elle dépend aussi de la place de l'établissement dans son environnement et des caractéristiques des lieux fréquentés par les adolescents. Les discours des éducateurs et des adolescents congruent dans un premier temps dans la prise en compte de l'extérieur comme un espace indifférencié, caractérisé par l'absence de regard des éducateurs sur les adolescents. Cependant, l'appréciation de la localisation des établissements et les remarques concernant leur accessibilité par les éducateurs montrent que le sens conféré à la mobilité dépend des caractéristiques attribuées à ce dehors.

Pour les éducateurs comme pour les adolescents, les déplacements autonomes à l'extérieur de l'établissement sont caractérisés par l'absence de regard des éducateurs sur les adolescents. En entretien, les éducateurs répondent par exemple difficilement aux questions concernant les lieux fréquentés par les adolescents lorsqu'ils ne sont pas présents, notamment lorsque la sortie est effectuée sans autorisation. Les sorties à l'extérieur de l'établissement sont alors considérées comme mettant potentiellement en péril la protection des adolescents et évoquées avec le terme de « mises en danger ». Ces risques sont avant tout liés à l'absence de surveillance que la présence des adolescents à l'extérieur de l'établissement entraîne, comme le révèlent ces extraits d'entretien :

La vérité on la saura jamais vraiment, quand elle [adolescente] est pas là elle est pas là (Entretien, éducateur Foyer 3, 2012)

- Quel est le rôle des sorties à l'extérieur dans le projet éducatif?

- Elles ont un rôle dans le sens où... déjà... comment on arrive à pouvoir échanger de manière authentique ou pas sur ce qui se passe dehors, ce qui se passe dehors pour elles [adolescentes]... Est-ce que dehors elles..., qui elles rencontrent, dans quelle mesure ce qu'elles font dehors est bon pour elles ou peut les mettre en danger [...]

(Entretien, éducateur Foyer 3, 2012) 
En contraste avec cette inquiétude, la mobilité est souvent synonyme de liberté pour les adolescents. L'extérieur représente en effet un espace de liberté car le jeune n'est pas tenu de référer aux adultes ce qui s'y déroule. Cette association entre espace extérieur et absence de regard des adultes est soulignée par plusieurs adolescents au cours d'entretiens. La difficulté d'obtenir, en tant qu'enquêtrice, des informations précises sur les lieux de sortie des jeunes est aussi révélatrice de la dimension secrète des espaces extérieurs et de la volonté de la préserver. L'un des adolescents interrogés a ainsi refusé de répondre à nos questions en invoquant le caractère « secret » des endroits qu'il pratique. Alors que nous insistons sur la confidentialité de l'entretien, il se montre particulièrement méfiant et ne répond pas (Entretien, adolescent Foyer 1, 2012).

Malgré cette inconnue, les éducateurs tentent dans certains cas de savoir où se rendent les adolescents pendant leurs sorties. Les témoignages des autres adolescents ou de l'adolescent concerné peuvent leur donner des pistes, même si elles sont toujours teintées d'incertitude. Dans ce contexte, l'imaginaire associé à certains lieux, eux-mêmes mal connus, est également source d'inquiétude pour les éducateurs. Ceux-ci évoquent par exemple « des endroits isolés, au bord de lac, des choses comme ça, c'est pas dans la ville, c'est isolé » (Entretien, éducateur Foyer 3, 2012) ou des quartiers dangereux, lorsqu'ils parlent des sorties des adolescents. Dans les deux cas, la dangerosité des espaces est liée à une réputation plus qu'à leur connaissance directe. À propos des «endroits isolés », un éducateur précise ainsi qu'il parle d'" une partie un peu plus glauque... tout le monde sait » (Entretien, éducateur Foyer 3, 2012). De même une éducatrice évoque « les quartiers » où elle n'est jamais allée elle-même mais dont des collègues lui ont parlé :

C'est vraiment un mode de vie différent. Moi je connais pas mais quand ils me l'expliquent et quand d'autres collègues me l'expliquent parce qu'ils connaissent aussi, ça n'a rien à voir avec ce que j'ai pu connaître. (Entretien, éducatrice Foyer 2, 2012)

Inconnus des éducateurs, et tenus secret par les adolescents, les lieux fréquentés lors des sorties autonomes sont principalement caractérisés par cette méconnaissance. Les représentations associées à ces lieux inconnus jouent alors un rôle dans la manière dont les éducateurs interprètent les déplacements des adolescents. Leur appréciation de la localisation de l'établissement montre des interprétations différenciées de la mobilité des adolescents en fonction de la destination supposée des déplacements qu'elle permet.

Interrogées sur le rôle de la localisation de l'établissement dans leur pratique éducative, les réponses de ces deux éducatrices montrent la dualité de la prise en compte de l'accessibilité, comme ressource ou contrainte, en fonction des adolescents, des modalités de leur sortie et des lieux qu'ils fréquentent :
C'est favorable et en même temps ça a un côté..., on n'est pas complètement perdus en campagne donc on peut pas se dire « de toute manière le gamin il peut pas fuguer parce qu'il va se retrouver vite tout seul sur la route ». Donc là en 5 minutes il est à [station de métro], alors ça peut être un atout pour les formations, pour tout ce qui est formation, scolarité, et puis même pour leurs sorties avec leurs amis je trouve que c'est agréable. Après ça peut être un inconvénient du fait qu'on soit trop proche de la ville et des tentations de la ville. (Entretien, éducatrice Foyer 4, 2013)

Donc même si on est un petit peu excentré du centre de Lyon, c'est suffisamment desservi par les transports pour qu'ils puissent accéder seuls à leur lieux de scolarisation, à leurs activités, à leurs familles. Voilà après le côté pervers de la chose c'est qu'ils sont peut-être, du fait justement de ces transports en commun qui desservent la zone, ben ils accèdent très facilement aussi à tous les lieux où ils peuvent se retrouver pour faire des bêtises. Donc notamment l'Opéra, le quartier de l'Opéra à Lyon qui est forcément reconnu pour accueillir nos petites bandes de jeunes qui font n'importe quoi. Voilà, la Part Dieu qui est leur lieu de prédilection, ils font rien mis à part zoner mais ils y vont quand même. (Entretien, éducatrice Foyer 2, 2012)

Ces extraits d'entretiens font apparaître des pratiques acceptées et même encouragées, en lien avec la scolarité, la formation, le maintien de liens familiaux et sociaux, au titre desquelles l'accessibilité de l'établissement est présentée comme une ressource. Elles sont mises en regard de pratiques hors-norme ( « fuguer », « zoner », « traîner », « faire n'importe quoi ») associées à des lieux particuliers, dont la mauvaise réputation est explicite (« le quartier de l'Opéra à Lyon qui est forcément reconnu pour accueillir nos petites bandes de jeunes ») ou implicite (la gare de Perrache est ainsi associée à la prostitution et à la consommation de drogue dans l'imaginaire collectif lyonnais). L'intégration des établissements à leur exosystème [24] est donc perçue de manière ambivalente par les éducateurs, en lien avec une double catégorisation des adolescents :

[L'impact de la localisation du foyer] dépend pour certains jeunes. Je dirais ça dépend des jeunes. Pour certains qui sont constamment dans la fuite ou en train de fuguer c'est un désavantage. Ils prennent la porte, ils sont rapidement dans le centre-ville, voilà, la localité elle permet de se déplacer facilement. Ça, ça peut être embêtant. Et pour certains autres jeunes, à qui on laisse des responsabilités ou quoique ce soit, c'est pratique. (Entretien, éducatrice Foyer 3, 2013)

Ici, la déviance juvénile n'est pas associée à un lieu particulier, mais au terme générique de « centre-ville » qui révèle la dimension essentiellement urbaine des pratiques hors-normes dans les représentations des éducateurs. La destination et l'objectif des déplacements des adolescents conditionnent la manière dont ils sont perçus par les éducateurs. La mobilité n'est alors pas considérée comme 
une compétence globale mais bien comme un moyen dont le sens ne peut être compris sans référence à un objectif précis. Or, les éducateurs ne peuvent pas toujours vérifier quels sont les lieux où se rendent les adolescents et sont donc plutôt méfiants face à leurs déplacements. Les différents extraits d'entretiens montrent également le rôle du rapport à l'espace des éducateurs dans le sens qu'ils attribuent à la mobilité des adolescents. En effet, leurs représentations des potentiels espaces fréquentés par les adolescents sont indissociables de leur propre parcours résidentiel et rapport à l'espace. L'éducatrice du Foyer 2 qui dit ne pas connaître le mode de vie des adolescents urbains est installée depuis un an à Lyon et insiste à plusieurs reprises lors de l'entretien sur son origine géographique et ses conséquences dans sa relation aux adolescents. Lorsque je lui demande si elle échange avec les adolescents sur les quartiers qu'elle évoque, elle répond :

Ils en parlent avec d'autres adultes qui connaissent justement ce mode de vie là. Moi ils m'en parlent en rigolant plus, en me disant, en me rappelant que c'est des choses que je ne connais pas et que je ne pourrai jamais comprendre, et je veux bien l'admettre en plus. Je vais pas avoir la prétention de leur dire que via des on-dit et des lectures je pourrais avoir une quelconque connaissance de ça. Donc ils partagent avec d'autres et ça c'est quelque chose sur lequel j'interviens du coup peu. Parce que pour eux, comme je disais, au-delà de vivre dans la campagne et dans la pampa, je viens d'un lieu idyllique et paradisiaque. (Entretien, éducatrice Foyer 2, 2012)

La relation entre le parcours résidentiel des éducateurs et le sens qu'ils accordent à la mobilité des adolescents nécessiterait d'être approfondie par une analyse plus systématique. Cependant, les éléments cités ici permettent d'émettre une première hypothèse quant aux différences observées au sein des éducateurs.

Au-delà de ces différences, les sens accordés à la mobilité par les éducateurs révèlent des divergences importantes par rapport aux sens objectivés, qu'ils fassent de la mobilité une norme sociale légitime ou au contraire un marqueur de marginalité. Pourtant, l'approfondissement de certaines questions ou les observations de terrain montrent que la mobilité est un enjeu important du placement. Les éducateurs font avec la mobilité des adolescents en même temps qu'ils l'impactent. La gestion des déplacements quotidiens s'inscrit ainsi dans un ensemble de conditions réglementaires et spatiales qui sont sans cesse négociées. Ces négociations révèlent les sens divergents accordés à la mobilité en fonction des groupes d'acteurs, éducateurs ou adolescents, mais elle s'inscrit globalement dans l'ambivalence de la mobilité à l'échelle de la société. Plus que la mobilité en elle-même, ce sont ses modalités et les lieux dont elle permet la fréquentation auxquels les éducateurs donnent un sens spécifique. Pourtant, l'enjeu de la mobilité dépasse cette dialectique et prend un sens propre au contexte institutionnel.

\section{La mobilité au prisme du placement, la construction d'un sens partagé propre à l'institution ?}

Dans certains cas, la mobilité est comprise de la même manière par les éducateurs et les adolescents et contribue à construire un sens commun propre à l'institution ${ }^{5}$. La mobilité est ainsi interprétée en lien avec la manière dont le placement est perçu par les adolescents. L'utilisation de termes spécifiques, comme celui de fugue, montre cependant que la construction de ce sens commun s'établit avec certaines nuances.

\subsection{La mobilité, enjeu dans la relation avec les éducateurs}

L'interprétation de la mobilité des adolescents est liée à la relation entre éducateurs et adolescents. Alors qu'elle intervient dans la relation de pouvoir entre adolescents et éducateurs, la mobilité permet dans le même temps d'évaluer cette relation. En retour, la relation adolescentéducateur elle-même influence les conditions de mobilité des adolescents.

La mobilité est tout d'abord un moyen de régulation des rapports de pouvoir entre éducateurs et adolescents. Le fait même de sortir dans le cas d'une sortie non autorisées constitue une transgression à la règle et signifie la non-soumission de l'adolescent à l'institution. Interrogés sur les règles du foyer concernant les sorties, plusieurs jeunes ont ainsi différencié les règles du foyer et leurs propres règles : « ils ont leurs règles, moi $\mathrm{j}$ 'ai les miennes ! 》 (Entretien, adolescente Foyer 3, 2012). La sortie non autorisée constitue donc une transgression particulière : signifiant la non-soumission du jeune au règlement, elle lui permet aussi de se soustraire au regard de l'adulte et éventuellement de réaliser d'autres activités interdites. Selon certains éducateurs, la transmission des possibilités pour sortir de l'établissement sans autorisation se fait dès l'arrivée d'un nouvel adolescent. Interrogé sur la fréquence des sorties sans autorisation, un éducateur précise ainsi à propos des jeunes filles placées dans cet établissement : « elles s'apprennent quasiment dès l'arrivée les unes aux autres comment on fait pour sortir » (Entretien, éducateur Foyer 3, 2012).

Si la sortie non autorisée est un moyen pour les adolescents de se soustraire aux règles de l'établissement, les sorties peuvent également signifier la conformité du comportement des adolescents aux attendus de l'établissement. Questionnée sur les possibilités de sortie sans adulte, L.

\footnotetext{
${ }^{5}$ Ce sens n'est pas exhaustif et la mobilité peut prendre d'autres sens notamment pour les adolescents [47].
} 
explique ainsi : «si t'es sage, tu sors » (adolescente, Foyer 4, 2013). Les autorisations de sortie sans adulte, comme les sorties avec les éducateurs, peuvent être considérées comme des récompenses par les adolescents et les adultes. Ainsi, un accompagnement ou l'organisation d'une sortie spécifique peuvent intervenir pour féliciter un bon comportement ou inciter un adolescent à entrer en contact avec les adultes.

Dans ce contexte, les pratiques des jeunes, et notamment le respect des règles de sortie, sont un moyen pour les éducateurs d'évaluer l'appropriation de la prise en charge par le jeune. La fréquence des fugues est notamment un indicateur de l'évolution du rapport d'un adolescent à la prise en charge, comme dans le cas de F. Pour cette jeune fille, la baisse de fréquence des fugues peut être mise en relation avec le temps de placement et le lien croissant entre elle et les éducateurs. La négociation de la règle est ici rendue possible car F. explique aux éducateurs le but de ses sorties, ce qui est perçu comme un progrès par les éducateurs, malgré le non-respect du règlement intérieur :

Le traitement de la fugue ça se fait sur le long terme, avec le lien. Le cas de F. montre bien que ça fonctionne, quand elle est arrivée, elle fuguait tout le temps, [...], maintenant c'est beaucoup moins souvent, et elle prévient, « ce soir je ne rentrerai pas, je vais chez une copine... » (Entretien, éducatrice Foyer 3, 2012)

Différents types de sorties non autorisées ont été identifiés par les éducateurs interrogés en entretien. Les typologies varient en fonction des éducateurs mais la mise en relation de la fugue avec l'appropriation de la mesure de placement par les jeunes a plusieurs fois été évoquée :

Après y a la fugue « je ne comprends pas pourquoi je suis ici, j'ai rien à faire ici, ma place est chez moi ». (Entretien, éducateur Foyer 2, 2012)

Le fait de ne pas pouvoir rester ici, le sens qu'elles donnent au placement [...], que le modèle qu'on leur propose ici est trop en décalage avec celui qu'elles connaissent. (Entretien, éducateur Foyer 3, 2012)

Je pense qu'à partir du moment où ils se sentent en sécurité, ils mettent en place des choses positives pour eux qui leur permettent de demander une autorisation et de l'avoir, donc de ne pas avoir à fuguer. (Entretien, éducateur Foyer 1, 2012)

On retrouve ce sens dans les paroles des adolescents qui expliquent leurs sorties sans autorisations par le fait qu'ils n'ont pas envie d'être au foyer ou qu'ils sont mieux ailleurs :

Que penses-tu des règles concernant les sorties ?

$\mathrm{Au}$ début je respectais parce que je savais pas où aller, ça changeait rien... Après j'ai connu ma copine, c'était plus bénéfique pour moi d'être là-bas qu'ici. J'ai commencé à ne pas respecter parce que c'était loin, c'était trop tard pour le métro.

Et quelles étaient les conséquences?

Même s'il y a des conséquences, du moment que moi je me sentais bien... des fois ici c'est pas trop ça, des fois ici ça se passe pas bien.

(Entretien, adolescente Foyer 3, 2012)

Enfin, le mode de relation entre l'éducateur et l'adolescent peut en retour influencer l'appréciation de la mobilité par les éducateurs. Un exemple de cette relation est issu d'une observation directe :

\begin{abstract}
Alors qu'il est prévu qu'il aille chez le coiffeur, O. demande à son éducateur de s'y rendre seul, en vélo. Il part malgré le refus de son éducateur. Lorsque nous partons en voiture à sa recherche, l'éducateur suppose qu'il n'est pas chez le coiffeur mais plutôt dans la « cité » de la commune. À notre arrivée chez le coiffeur, le vélo est bien devant et $\mathrm{O}$. à l'intérieur. Je lance « Au moins, il a un bon sens de l'orientation ! », ce à quoi l'éducateur répond : «Ah ben oui, ça c'est sûr il est débrouillard, mais c'est dommage que ça puisse pas être basé sur de la confiance » (Carnet de terrain, Foyer 1, 2011)
\end{abstract}

S'il y a bien reconnaissance de la compétence de l'adolescent (sa débrouillardise), celle-ci est dévalorisée par l'absence de confiance, et donc la nécessité de surveillance, de l'éducateur vers l'adolescent.

Les modalités de sortie à l'extérieur de l'établissement sont donc au centre de la relation entre éducateurs et adolescents. Elles sont interprétées comme des signes d'acceptation ou de refus du placement et constituent en même temps des outils éducatifs : les comportements des adolescents sont ainsi sanctionnés via l'incitation aux sorties ou à leur répression. Les interprétations de la mobilité dans ses liens à la relation éducateur-adolescent semblent être partagées par les deux types d'acteurs. Ici, l'interprétation de la mobilité renvoie aux relations de pouvoir entre acteurs à l'intérieur du foyer. Le fait que les déplacements à l'extérieur puissent être favorisés ou limités en fonction de ces rapports de pouvoir invite à réfléchir à la relation entre liberté et mobilité [48]. La construction de ce sens commun de la mobilité, propre au contexte de placement, peut être interrogée à travers l'exemple du recours au terme de fugue.

\subsection{Un sens commun en construction : la fugue}

L'utilisation du terme fugue pour faire référence aux sorties réalisées en dehors du règlement du foyer, autant par les éducateurs que les adolescents, interroge ce sens commun. Le terme fugue a plusieurs sens selon les domaines envisagés. Au sens juridique, la fugue désigne 
« le fait pour le mineur de s'absenter d'un lieu où il est censé se trouver, et plus spécifiquement de se soustraire à l'autorité de son représentant légal ou de son gardien (parents, institution...) » [49]. Dans le domaine de la psychologie, il fait référence à une fuite et se distingue de l'« errance » par son caractère ponctuel, les fugues dites « initiatiques » pouvant par la suite se transformer en « errance » [50]. Ce dernier terme est aussi utilisé par les travailleurs sociaux mais n'est pas mentionné par les adolescents. Le terme de fugue, dont la complexité n'est ici qu'effleurée, est utilisé fréquemment par les différents acteurs des institutions de placement et signifie globalement toute sortie sans autorisation, pour n'importe quelle durée. Ce vocabulaire semble particulièrement ancré, autant chez les adolescents que chez les éducateurs. Ainsi, autant les uns que les autres ont mentionné en entretien ce terme en répondant à la question relative aux sorties non autorisées et en précisant d'emblée que " ça s'appelle une fugue ! » (Entretien, éducatrice Foyer 4, 2013). Le sens commun de la mobilité semble cristallisé par l'usage de ce terme unique par l'ensemble des acteurs. Il renvoie également à un sens de la mobilité centré sur l'institution : l'idée de fuite sous-entend en effet une prise en compte du déplacement depuis le lieu de placement vers l'extérieur. Si la destination et les objectifs du déplacement ont bien un rôle dans le sens que les éducateurs confèrent à la mobilité des adolescents, celle-ci est prise en compte de façon secondaire. On peut émettre l'hypothèse qu'en l'absence de certitude sur les lieux effectivement fréquentés par les adolescents, les éducateurs sont contraints à envisager le fait que les adolescents partent de l'établissement plutôt que le fait qu'ils aillent vers une destination.

Le sens donné aux fugues renvoie plus largement à une représentation de l'espace extérieur indifférencié et exerçant une attractivité sur les adolescents. Bien qu'il ne soit pas clairement défini, cet espace extérieur représente un risque pour les éducateurs. Le risque est essentiellement lié au fait que les adolescents ne semblent pas être en mesure de lutter contre la supposée attraction de l'espace extérieur et de ses tentations. En effet, la distance, liée à la localisation de l'établissement ou à la rupture des liens de communication est la solution préconisée par les éducateurs pour limiter cette attraction. La distance avec ces espaces d'attraction permet ainsi de limiter le risque. Cette distance est liée à la localisation du foyer mais concerne aussi l'ensemble des moyens de communication et notamment les téléphones portables et renvoie donc à une acception large des «systèmes de mobilité » [25] :

Ils sont pas trop parasités par l'environnement extérieur, donc ça permet aussi peut-être à certains de se poser beaucoup plus facilement et d'être moins happés par... ben par les bandes de djeuns qui pourraient les influencer dans tels ou tels trucs ou de vouloir constamment sortir et zoner dehors jusqu'à pas d'heure, là bon y a pas grand-chose à faire dans les environs. (Entretien, éducatrice Foyer 4, 2013)

C'est potentiellement, c'est être happé par l'extérieur. On n'a pas toujours le regard sur cet extérieur là donc ça peut être dangereux. Ça peut être des mises en danger, la grosse... le gros risque il est à ce niveau là. Si un gamin il est dans des comportements addictifs, le téléphone peut être simplement un outil de ça. C'est gênant et puis en pratique on n'a... à partir du moment où on n'a plus les gamins sous les yeux, ils peuvent se mettre en danger, on peut pas faire grand-chose. Bien sûr on peut pas tout maîtriser mais ça fait partie des points qui peuvent servir à attirer un gamin vers l'extérieur. (Entretien, éducateur Foyer 1, 2012)

- Donc y a un effet de l'éloignement?

- Je pense oui, du coup après ils se voient moins, parce qu'après un week-end sur deux ça fait juste, si y a pas de portable, ils peuvent pas s'appeler souvent donc le contact est moins..., y a pas de tentations dans les bêtises. (Entretien, éducatrice Foyer 1, 2012)

Dans ces trois extraits d'entretiens, la référence à un espace extérieur attractif (« happer », « attirer », « tentations ») suggère une distinction forte entre le foyer comme lieu et l'espace extérieur. Cette interprétation des déplacements par les éducateurs correspond à la forme de mobilité « sédentaire » [7], puisque toute expérience s'effectue en référence à un lieu précis. Les interprétations des fugues par les éducateurs font pourtant intervenir des causes endogènes et/ou exogènes. Ainsi, la fugue peut être liée à une envie de sortie dont le jeune sait qu'elle va lui être refusée, à une situation d'énervement, ou à un appel de l'extérieur (Entretien éducatrice Foyer 4, 2013). De ces trois explications, seule la deuxième relève vraiment de la fuite. La première et la troisième donnent à la fugue des causes exogènes, sans pour autant remettre en cause le terme. D'autres éducateurs comprennent la fugue uniquement comme une réaction de fuite de l'établissement ou d'évitement d'un potentiel refus de la demande d'autorisation de sortie : «Ben c'est soit de la fuite, soit qu'elles n'ont pas envie de se confronter au non. Parfois elles passent même pas par le bureau parce qu'elles savent qu'on va dire non! » (Entretien, éducatrice Foyer 3, 2012). D'autres encore mettent l'accent sur la fonction de la fugue dans la relation avec les éducateurs (un appel au secours) et sur des causes exogènes (la tentation du dehors, l'appel de la rue, l'appel des copains) (Entretien, éducatrice Foyer 2, 2012). L'usage unanime de ce terme par les adolescents et les éducateurs renvoie donc à des interprétations multiples, qui diffèrent d'un individu à l'autre.

Cette ambiguité induit des difficultés et malentendus dans le quotidien de la prise en charge, dont rend compte cet extrait de carnet de terrain. Parlant d'une situation complexe donnant lieu à des malentendus, une éducatrice explique ainsi : " c'est une fugue et pas une fugue, on sait pas 
trop... » (Carnet de terrain, Foyer 3, 2012). Cette ambiguité apparaît aussi dans l'expression utilisée par un éducateur qui, répondant à la question d'une collègue demandant si une adolescente est en fugue, lui répond qu'elle est en « fugue familiale» (Carnet de terrain, Foyer 2, 2012).

L'ambiguité de ce terme est notée par le psychologue d'un établissement qui préfère dire " ils vont chez papa/maman » plutôt qu' « ils fuguent ». En utilisant une périphrase, il met l'accent sur la volonté d'aller quelque part plutôt que sur la fuite d'un lieu. Le point de vue sur ce déplacement est alors complètement inversé, puisque la fuite devient en fait un retour. La nécessité de clarifier le sens du terme est aussi un des objectifs de la réunion consacrée à la fugue organisée dans un des établissements. Dans le cadre d'une réflexion sur l'établissement et l'évaluation de son action, « la fugue » est mise au programme d'une des réunions. À cette occasion, la psychologue de l'établissement souligne notamment la nécessité d'aller au-delà d'une catégorisation des adolescents comme fugueurs et de comprendre les motivations de la fugue. À la question d'une éducatrice sur l'attitude à avoir lors d'un retour de fugue, elle répond que « cela dépend de ce qui est fait pendant la fugue » (Carnet de terrain, Foyer 2, 2012). Cette réponse souligne l'intérêt d'une prise en compte plus fine de la fugue pour pouvoir en comprendre le sens.

L'usage et l'affirmation volontaire du terme fugue par les éducateurs et les adolescents semblent confirmer l'existence d'un sens de la mobilité commun et propre à l'institution. En effet, la qualification de certains déplacements de fugue est conditionnée par le contexte institutionnel et ne prendrait pas ce nom dans un autre contexte. Cependant, les sens donnés au terme fugue lui-même révèlent une multitude d'interprétations qui vont bien au-delà de la fuite du lieu de vie. Les réflexions individuelles de certains professionnels sont relayées par des initiatives institutionnelles, comme des réunions consacrées à la question des fugues, qui contribuent à clarifier et uniformiser ces sens en construction.

En conclusion, la mise en regard des différents sens de la mobilité dans la société actuelle, objectivés par la littérature scientifique, et des sens construits par les différents acteurs de l'institution, éclaire la construction dynamique du sens de la mobilité des adolescents dans le contexte de placement. Le sens donné à la mobilité est ainsi replacé dans les différents cadres dans lesquels l'individu en développement évolue [24], et notamment le contexte institutionnel. Les sens de la mobilité au sein du microsytème « foyer » entrent en écho avec l'ambivalence de la mobilité à l'échelle de la société. On retrouve, autant au niveau du microsystème que du macrosystème, des sens de la mobilité construits selon un ensemble de normes. La prise en compte de la mobilité comme valeur de la société peut ainsi être nuancée : le sens accordé par les éducateurs à la mobilité des adolescents placés souligne la possible connotation négative de la mobilité, lorsque sa réalisation dans le déplacement n'est pas conforme aux normes sociales.

Malgré ces analogies entre les sens de la mobilité au niveau de la société et de l'institution de placement, la construction du sens de la mobilité dans le contexte de placement présente certaines spécificités. La mobilité comme compétence à favoriser, que l'on retrouve dans le champ scientifique comme dans les politiques publiques, apparaît finalement peu dans les entretiens. Il ne s'agit donc pas d'un enjeu explicite, mais plutôt d'un impensé de la prise en charge. La mobilité n'en est pas moins un enjeu fort du placement, que cette analyse met en lumière. Impensé, le sens de la mobilité apparaît dans la manière dont sont gérés les déplacements des adolescents et dans les représentations que les éducateurs ont de l'établissement compris dans son environnement. Les enjeux se déclinent ainsi à l'échelle micro, celle du franchissement de la limite entre l'intérieur et l'extérieur de l'établissement, et à une échelle plus large, en prenant en compte les destinations des déplacements. En effet, bien que les éducateurs ne puissent pas toujours savoir où se rendent les adolescents lorsqu'ils sortent seuls, leurs représentations des lieux supposément fréquentés intervient dans la construction des sens de la mobilité et notamment de son appréciation positive ou négative. Enjeu conflictuel entre éducateurs et adolescents, la mobilité est interprétée au prisme du placement aussi bien par les premiers que par les seconds. Le rôle de la sortie dans la relation éducative est par exemple un sens propre aux institutions de placement. Il est compris de manière commune par les éducateurs et les adolescents et indépendamment d'une référence au sens social de la mobilité. L'exemple du terme fugue, utilisé et revendiqué par les deux types d'acteurs, montre bien ce sens propre de la mobilité. Lié au cadre et à la réglementation des sorties à l'extérieur de l'établissement, ce terme unique est cependant nuancé par des interprétations multiples aux sens parfois eux-mêmes divergents.

\section{Bibliographie}

1. Bourquin J (2007) Le mineur de justice : enfance coupable, enfance victime ? Revue d'histoire de l'enfance "irrégulière 》 Hors-série. http://rhei.revues.org/3011

2. Sauvadet T (2006) Jeunes dangereux, jeunes en danger : comprendre les violences urbaines. Dilecta, Paris.

3. Pédron P (2005) Droit et pratique de la Protection Judiciaire de la Jeunesse, Mineurs en danger, mineurs délinquants. Gualino Éditeur, Paris.

4. Lévy J, Lussault M (2013) Dictionnaire de la géographie et de l'espace des sociétés. Belin, Paris.

5. Brunet R, Ferras R, Théry H (1993) Les mots de la géographie : dictionnaire critique. Reclus, La documentation française, Paris.

6. Kaufmann V (2006) Motilités, latence de mobilité et modes de vie urbains. In : Bonnet M, Aubertel P (eds.) La ville aux limites de la mobilité, Paris, PUF, Paris, pp 223-233.

7. Montulet B (2005) Au-delà de la mobilité : des formes de mobilités. Cahiers internationaux de sociologie 1/118: 137-159. DOI : $10.3917 /$ cis. 118.0137 
9. Boltanski L, Chiapello È (1999) Le nouvel esprit du capitalisme. Gallimard, Paris.

10. Massot M-H, Orfeuil J-P (2005) La mobilité au quotidien, entre choix individuel et production sociale. Cahiers internationaux de sociologie 118(1) : 81-81 DOI : 10.3917/cis.118.0081

12. Cresswell $\mathrm{T}$ (2004) Justice sociale et droit à la mobilité. In : Allemand S, Ascher F, Lévy J (eds.) Les sens du mouvement. Belin, pp 145-153.

13. Roméro C, Dumont M (2008) Les jeunes dans leur mobilités. L'espace en tous sens. In : Séchet R, Garat I, Zeneidi D (eds.) Espaces en transactions. Presses Universitaires de Rennes, Rennes. pp 109-120.

13. Brachet J (2012) Géographie du mouvement, géographie en mouvement. La mobilité comme dimension du terrain dans l'étude des migrations. Annales de géographie 5: 543-560.

14. Beaud S (1996) L'usage de l'entretien en sciences sociales. Plaidoyer pour 1'《 entretien ethnographique ». Politix 9(35) : 226-257.

15. Morelle M, Ripoll F (2009) Les chercheur-es face aux injustices : l'enquête de terrain comme épreuve éthique. Annales de géographie 665-666 : 157-168 DOI : 10.3917/ag.665. 0157

16. Marcelli D (2010) Adolescence. In : Le Breton D, Marcelli D. (eds.) Dictionnaire de l'adolescence et de la jeunesse. PUF, Paris, pp 15-19.

17. Massot M-H, Zaffran J (2007) Auto-mobilité urbaine des adolescents franciliens. Espace Populations Sociétés 2-3 : 227-241.

18. Depeau S (2003) L'enfant en ville : autonomie de déplacement et accessibilité environnementale. Thèse de psychologie sociale et environnementale, Université René Descartes, Paris 5.

19. Kaufmann V, Widmer E (2005) L'acquisition de la motilité au sein des familles. Espaces et sociétés 2 : 199-217.

20. Depeau S (2008) Radioscopie des territoires de la mobilité des enfants en milieu urbain. Comparaison entre Paris intra-muros et banlieue parisienne. Enfances, Familles, Générations 8, http://id.erudit.org/iderudit/018489ar.

21. Oppenchaim N (2009) Mobilités quotidiennes et ségrégation : le cas des adolescents de Zones Urbaines Sensibles franciliennes. Espace, populations, sociétés $2: 215-226$.

22. Boujut S, Fréchon I (2009) Inégalités de genre en protection de l'enfance. Revue du Droit sanitaire et social 6 : 1003-1015.

23. Masson B (2010) « Mineurs isolés étrangers »: le sens d'une appellation. Migrations Sociétés 22(129-130) : 115-128.

24. Bronfenbrenner U (1977) Toward an Experimental Ecology of Human Development. American Psychologist 32: 513-531.

25. Urry J (2005) Les systèmes de la mobilité. Cahiers internationaux de sociologie 118(1) : 23-35 DOI : 10.3917/cis.118.0023

26. Orfeuil J-P (2011), Dix ans de « droit à la mobilité », et maintenant? Métropolitiques, http://www.metropolitiques.eu/Dixans-de-droit-a-la-mobilite-et.html

27. Orfeuil J-P (2010), La mobilité, nouvelle question sociale ? SociologieS http://sociologies.revues.org/3321

28. Lannoy P, Ramadier T (2007) La mobilité généralisée : formes et valeurs de la mobilité quotidienne. Bruylant-Academia, Louvainla-Neuve.

29. Bacqué M-H., Fol S (2007) L'inégalité face à la mobilité : du constat à l'injonction. Revue Suisse de Sociologie 33 : 89-104.

30. Authier J-Y (2006) La question des « effets de quartier » en France. Variations contextuelles et processus de socialisation. In : Authier J-Y, Bacqué M-H, Guérin-Pace F (eds.) Le quartier. Enjeux scientifiques, actions politiques et pratiques sociales. La Découverte, Paris, pp 206-216.
31. Séchet R, Garat I, Zeneidi D (2008) Introduction. In : Séchet R, Garat I, Zeneidi D (eds.) Espaces en transactions. Presses Universitaires de Rennes, Rennes, pp 7-26.

32. Ripoll F, Veschambre V (2005) Sur la dimension spatiale des inégalités : contribution aux débats sur la «mobilité et le capital spatial ». In : Arlaud S, Jean Y, Royoux D (eds.) Rural-urbain, Nouveaux liens, nouvelles frontières. Presses universitaires de Rennes, Rennes. pp 467-483.

33. Veschambre V (2006) Penser l'espace comme une dimension de la société. Pour une géographie sociale de plain-pied avec les sciences sociales. In : Séchet R, Veschambre V (eds.) Penser et faire la géographie sociale. Contributions à une épistémologie de la géographie sociale. Presses Universitaires de Rennes, Rennes. pp 211-227.

34. Cresswell T (1996) In placelout of place: geography, ideology, and transgression. University of Minnesota Press, Minneapolis, London.

35. Lussault M (2009) De la lutte des classes à la lutte des places. Grasset, Paris.

36. Jouffe Y (2007) Précaires mais mobiles. Tactiques de mobilité des travailleurs précaires flexibles et nouveaux services de mobilité. Thèse de Sociologie, École Nationale des Ponts et Chaussées.

37. Fol S (2010) Mobilité et ancrage dans les quartiers pauvres : les ressources de la proximité. Regards Sociologiques 40:27-43.

38. Coutard O, Dupuy G, Fol S (2002) La pauvreté périurbaine : dépendance locale ou dépendance automobile ? Espaces et sociétés 108(1) : 155-176.

39. Retière J-N (2003) Autour de l'autochtonie. Réflexions sur la notion de capital social populaire. Politix 16(63) : 121-143.

40. Rénahy N (2010) Classes populaires et capital d'autochtonie Genèse et usages d'une notion. Regards sociologiques 40 : 9-26.

41. Bonerandi-Richard E (2014) La pauvreté masquée des espaces ruraux français : analyses locales en Thiérache et dans l'Ain. In : Boulineau E, Bonerandi-Richard E (eds.) La pauvreté en Europe. Une approche géographique. Presses Universitaires de Rennes, Rennes. pp 101-134.

42. Vignal C (2005) Les espaces familiaux à l'épreuve de la délocalisation de l'emploi : ancrages et mobilités de salariés de l'industrie, Espaces et sociétés 2(120-121) : 179-197 DOI : 10.3917/esp.120.0179

43. Le Breton E (2005) Bouger pour s'en sortir. Mobilité quotidienne et intégration sociale. Armand Colin, Paris.

44. Gachelin M (2007) Les jeunes et la mobilité. EMPAN 67 : 60-64.

45. Zeneidi D (2010) Jeunesse et errance. La construction d'une altérité problématique. In : Danic I, David O, Depeau S (eds.) Les enfants et les jeunes dans les espaces du quotidien. Presses Universitaires de Rennes, Rennes, pp 221-232.

46. Damon J (2004) (Im)mobilité(s) et pauvreté(s). In : Orfeuil J-P (eds.) Transports, pauvretés, exclusions. Pouvoir bouger pour s'en sortir. Éditions de l'aube, La Tour d'Aigues, pp 103-111.

47. Guy F (2013) «J'habite... enfin..., je me comprends »: l'appropriation territoriale des adolescents placés en question. Enfances, Familles, Générations 19: 127-144.

48. Sheller M (2008) Mobility, Freedom and Public Space. In : Bergmann S, Sager T (eds.) The ethics of mobilities: rethinking place, exclusion, freedom and environment. Ashgate, Aldershot, Burlington, pp 25-38.

49. Groupe de travail (2004) Fugues, enlèvements, disparitions de mineurs, Mission confiée par Monsieur Dominique Perben, garde des Sceaux, ministre de la Justice et Monsieur Christian Jacob, ministre délégué à la Famille.

50. Chobeaux F (2004) Les nomades du vide. La Découverte, Paris. 